\title{
Power Flow for a Four-Wire Radial Low Voltage Distribution Grid with a Single Point Grounded Neutral
}

\author{
Andreas Kotsonias, Lenos Hadjidemetriou, Elias Kyriakides \\ KIOS Research and Innovation Center of Excellence and Department of Electrical and Computer Engineering \\ University of Cyprus \\ Nicosia, Cyprus \\ Email: \{kotsonias.andreas, hadjidemetriou.lenos, elias\}@ucy.ac.cy
}

\begin{abstract}
The forward/backward sweep is the most commonly used power flow method in radial Low Voltage Distribution Grids (LVDGs). In most cases, Kron's reduction is used to merge the neutral conductor with the phase conductors. However, important information can be lost by ignoring the effect of the neutral conductor through the Kron's reduction method, since in LVDGs the majority of loads are single-phase connected and are supplied through a phase conductor and the neutral. In this paper, a modification to the forward/backward sweep method is proposed to account for the neutral voltage as well. Test results indicate the significance of knowing the exact configuration of the neutral conductor in an LVDG before conducting power flow studies as it can affect greatly the accuracy of the results.
\end{abstract}

Index Terms - four wire system, low voltage distribution grid, neutral conductor, power flow, radial grid.

\section{INTRODUCTION}

The increasing share of the solar energy in the energy mix by residential rooftop photovoltaic (PV) systems and the eventual electrification of the transportation sector with electric vehicles $(E V s)$ are some of the main reasons behind the unprecedented technical growth that has been undergoing over the past few years in LVDGs [1]. These advancements are creating new challenges to the Distribution System Operators (DSOs) regarding the secure and reliable operation of their networks [2]. For this reason, it is important to have accurate and reliable tools to conduct studies, identify potential problems and proactively mitigate them before costly faults or equipment failures occur. The most common tool used for this kind of studies is the power flow analysis which, for a given set of input parameters, can provide the magnitudes and angles of all voltages and currents of a power system. The traditional GaussSeidel and Newton-Raphson power flow methods are focused on a balanced operation which allows the analysis to be conducted only on the positive sequence. On the contrary, distribution systems, especially LVDGs, are characterized by highly unbalanced operating conditions. As a consequence, the negative and even the zero sequence components are not negligible and therefore must be accounted for by using multiphase analysis. Currently, the main methods used for solving the distribution grid power flow problem are either focused on the current injection technique which is based on the NewtonRaphson formulation [3] or on the Forward/Backward Sweep (FBS) method [4]. While the current injection method is more robust and can be even applied in large meshed networks, the FBS method has been established as a preferable solution for radial LVDGs due to the simplicity and computational advantage of the method [5].

FBS is an iterative method and it can be divided to two parts. In the first part (backward sweep), all the load, line and branch currents are calculated using the known PQ values and an initial guess for the voltage (flat start). In the second part (forward sweep), the system voltages are updated, starting from the reference node, by using the calculated currents, from the first part, and the impedance matrix. Convergence is achieved when the voltage update $\Delta V$ from one iteration to the next is lower than a predefined limit $\varepsilon(\|\Delta V\|<\varepsilon)$. In [6], an algorithm is proposed that can implement FBS by using only the conventional bus-branch oriented data to construct two matrices which are used to solve the power flow problem directly. A similar approach is followed in [7], where the authors also consider and incorporate different load connections, distribution transformers and shunt capacitances. In [8], distributed generators are also considered after slightly modifying the FBS method so the nodes in which they are installed are treated as PV nodes instead of PQ nodes. A modified FBS algorithm is proposed in [9] which introduces three matrices $\mathrm{A}, \mathrm{B}$ and $\mathrm{C}$ in order to easily identify downstream buses and to calculate branch currents. This method is found to converge in slightly less iterations than the method in [6]. In [10] the FBS method is modified and is applied to a three-phase, four-wire, multi-grounded radial distribution network. It should be noted that in [6]-[9] the neutral conductor was either neglected completely or it was merged with the phase conductors with the use of the Kron's reduction method. However, this requires either a multigrounded neutral conductor or that the neutral voltage can be approximated to zero. By using Kron's reduction method all information regarding the operational state of the neutral conductor is lost. In [10], despite assuming a multi-grounded neutral conductor, the Kron's reduction method is not applied 
and therefore the power flow results include also the neutral voltage and current.

Due to the unbalanced nature of the LVDG and the mainly single-phase loads (asymmetric loading conditions), a significant portion of the total load current flows in the neutral conductor. Especially in a system that the neutral conductor is only grounded at the MV-LV transformer substation, this gives rise to a significant voltage drop across it [11]. By using Kron's reduction to simplify the problem and merge the neutral conductor with the phase conductors not only will yield incorrect results [12] but also important information will be lost as most loads in these kinds of systems are connected between a phase conductor and the neutral conductor. This paper takes inspiration by [10] and extends it so that it can be applied in LVDGs with a neutral conductor grounded only at the MV-LV transformer substation. Additionally, comparisons are made between the conventional approach of using Kron's reduction $\left(F B S_{C}\right)$, the FBS described in [10] $\left(F B S_{N g}\right)$ and the proposed one $\left(F B S_{N}\right)$. The results indicate a significant increase in accuracy and robustness which points out the significance of knowing the exact configuration of the neutral conductor before conducting power flow studies in LVDGs. The paper is organized as follows. In Section II the relevant key elements of the $F B S_{N}$ are presented along with the proposed modifications. In Section III, the $F B S_{C}, F B S_{N g}$ and $F B S_{N}$ are applied to an LVDG with a neutral conductor grounded only at the MV-LV transformer substation for evaluating the effectiveness of each method. Finally, the paper concludes in Section IV.

\section{FORWARD BACKWARD SWEEP METHOD}

In Fig. 1 the general algorithm of the FBS methodology is illustrated. At first, the conventional bus-branch oriented data of the LVDG are loaded so that the topology of the network can be constructed. Fig. 2 illustrates a typical 4-wire distribution line connecting two system nodes. Due to the asymmetric operating conditions, the mutual impedances cannot be merged with the series impedances and therefore such a line is characterized by a $4 \times 4$ matrix,

$$
Z_{i j}=\left[\begin{array}{llll}
Z_{a a} & z_{a b} & Z_{a c} & Z_{a n} \\
Z_{b a} & Z_{b b} & Z_{b c} & Z_{b n} \\
Z_{c a} & Z_{c b} & z_{c c} & Z_{c n} \\
Z_{n a} & z_{n b} & Z_{n c} & Z_{n n}
\end{array}\right]
$$

Each element of the impedance matrix is calculated using the available conductor data, the geometry of the distribution lines and the Carson's equations [13]. In $F B S_{C}$ the Kron's reduction method is used to merge the neutral conductor with the phase conductors, reducing the size of the matrix to $3 \times 3$.

\section{A. Flat Start}

In the first iteration of the algorithm all system voltages are initialized according to the voltage of the reference node. In [6][10] the step-down transformer was neglected from the simulation studies and the low voltage side of the transformer was selected as the reference node with balanced voltages. However, due to unbalanced operation of the LVDG this is not recommended as even this node may have considerable voltage asymmetry which can affect all the downstream nodes. In this paper, the reference node is the primary side (medium voltage) of the MV-LV transformer. The most common step-down

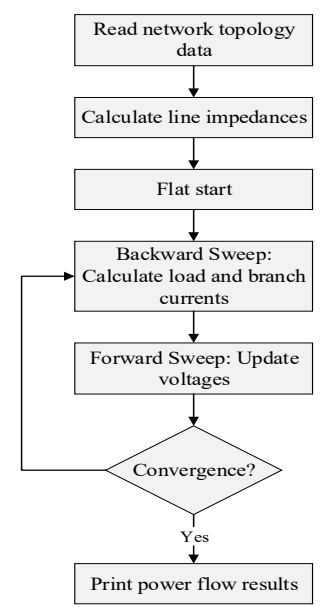

Figure 1. FBS general methodology

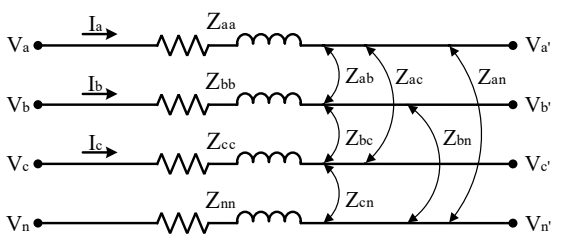

Figure 2. A four-wire distribution line

transformer configuration in LVDGs is D- $\mathrm{Y}_{\mathrm{g}}$ and its model can be found in [14]. By assuming an American standard configuration (positive sequence quantities on the high voltage side lead their corresponding quantities on the low voltage side by $30^{\circ}$ ) the system voltages in the LVDG are initialized as,

$$
\left[\begin{array}{c}
V_{a} \\
V_{b} \\
V_{c} \\
V_{n}
\end{array}\right]^{(0)}=\left[\begin{array}{c}
\left|V_{\text {ref }}\right| \measuredangle-30^{\circ} \\
\left|V_{\text {ref }}\right| \measuredangle-150^{\circ} \\
\left|V_{\text {ref }}\right| \measuredangle 90^{\circ} \\
0
\end{array}\right]
$$

\section{B. Backward Sweep}

In each iteration during the backward sweep, first, the load currents are calculated using the scheduled power injections and the voltages from the previous iteration. In [6]-[10], the load currents are calculated using the scheduled power and the corresponding phase voltage. However, in an LVDG a singlephase load is usually supplied by a phase conductor and the neutral conductor. Additionally, due to having the neutral conductor grounded only at the MV-LV substation, its voltage can reach significant values, especially towards the end of the LVDG. Therefore, during the calculation of the load currents in the backward sweep, the neutral voltage should be properly considered according to,

$$
\left[\begin{array}{c}
I_{a}^{i} \\
I_{b}^{i} \\
I_{c}^{i} \\
I_{n}^{i}
\end{array}\right]^{k}=\left[\begin{array}{l}
\left(\frac{S_{a}^{i}}{\left(V_{a}^{i}-V_{n}^{i}\right)^{k-1}}\right)^{*} \\
\left(\frac{S_{b}^{i}}{\left(V_{b}^{i}-V_{n}^{i}\right)^{k-1}}\right)^{*} \\
\left(\frac{S_{c}^{i}}{\left(V_{c}^{i}-V_{n}^{i}\right)^{k-1}}\right)^{*} \\
-\left(I_{a}^{i}+I_{b}^{i}+I_{c}^{i}\right)^{k}
\end{array}\right]
$$




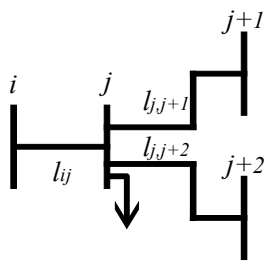

Figure 3. Section of a distribution grid

where $k$ denotes the iteration number, $i$ is the $i^{\text {th }}$ node with a load, $S_{a}^{i}, S_{b}^{i}$ and $S_{c}^{i}$ is the scheduled power of this load and $V_{a}^{i}$, $V_{b}^{i}, V_{c}^{i}$ and $V_{n}^{i}$ are the voltages of this node.

Having the load currents, then the branch currents must be calculated with the use of the network topology. In Fig. 3 a section of distribution grid is illustrated. The branch current in line $l_{i, j}$ can be calculated as,

$$
\left[\begin{array}{c}
I_{a}^{i, j} \\
I_{b}^{i, j} \\
I_{c}^{i, j} \\
I_{n}^{i, j}
\end{array}\right]^{k}=\left[\begin{array}{c}
I_{a}^{j} \\
I_{b}^{j} \\
I_{c}^{j} \\
I_{n}^{j}
\end{array}\right]^{k}+\sum_{m \in M}\left[\begin{array}{c}
I_{a}^{j, m} \\
I_{b}^{j, m} \\
I_{c}^{j, m} \\
I_{n}^{j, m}
\end{array}\right]^{k}
$$

where $I^{i, j}$ is the current flow in line $l_{i, j}, I^{j}$ is the current injection at node $j$ and $M$ is a set of the downstream nodes that node $j$ is connected to $(M=j+1, j+2)$.

\section{Forward Sweep}

During the forward sweep, the calculated branch and load currents from the backward sweep are used to update the system voltages, starting from the reference node. From Fig. 3, after the voltages at node $i$ are updated, the voltages at node $j$ are updated as,

$$
\left[\begin{array}{c}
V_{a}^{j} \\
V_{b}^{j} \\
V_{c}^{j} \\
V_{n}^{j}
\end{array}\right]^{k}=\left[\begin{array}{c}
V_{a}^{i} \\
V_{b}^{i} \\
V_{c}^{i} \\
V_{n}^{i}
\end{array}\right]^{k}-\left[\begin{array}{llll}
Z_{a a} & z_{a b} & Z_{a c} & Z_{a n} \\
Z_{b a} & Z_{b b} & z_{b c} & Z_{b n} \\
Z_{c a} & z_{c b} & Z_{c c} & Z_{c n} \\
z_{n a} & z_{n b} & z_{n c} & z_{n n}
\end{array}\right]_{l_{i j}}\left[\begin{array}{c}
I_{a}^{i, j} \\
I_{b}^{i, j} \\
I_{c}^{i, j} \\
I_{n}^{i, j}
\end{array}\right]^{k}
$$

Equation (5) is repeated until the voltages of all system nodes are updated.

\section{Convergence}

After all voltages have been updated in the $k^{\text {th }}$ iteration, the voltage step $\Delta V$ is calculated as,

$$
\Delta V^{k}=\left|V^{k}-V^{k-1}\right|
$$

where $V^{k}$ is a vector with all node voltages from the $k$ iteration and $\Delta V^{k}$ is also a vector with the voltage step of each system voltage from the $k-1$ iteration to the $k$. If any voltage step exceeds a pre-defined limit $\varepsilon$, then the process is repeated until (7) is satisfied.

$$
\left\|\Delta V^{k}\right\|_{\infty}=\max \left\{\Delta V^{k}\right\}<\varepsilon
$$

\section{CASE STUDIES}

In this section the $F B S_{C}, F B S_{N g}$ and $F B S_{N}$ are applied for power flow studies for the LVDG that is illustrated in Fig. 4.

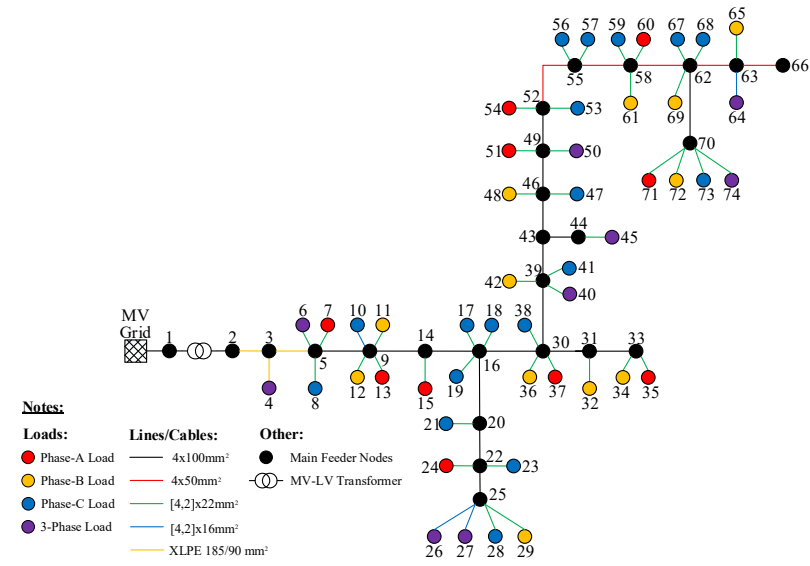

Figure 4. The LVDG under consideration

This is an actual LVDG in the power system of Cyprus and it serves mainly single-phase loads and a small number of threephase loads. Additionally, the neutral conductor is only grounded at the $11 \mathrm{kV} / 433 \mathrm{~V}$ MV-LV transformer substation. The system model is also implemented in MATLAB/Simulink and its power flow results are compared to the results of each method in order to determine their accuracy.

\section{A. Power Flow Results}

Initially, each system load is assigned a random value for its active power from the interval $0.1-4 \mathrm{~kW}$ and a random power factor from the interval $0.93-0.99$ lagging. In Fig. 5 the error in the calculation of the voltage magnitude of each node (average value of the phase voltages error) is illustrated for all three methods. It can be seen from this figure that using Kron's reduction $\left(F B S_{C}\right)$ in such a system will yield inaccurate results regarding the operational voltage of the LVDG. Additionally, by including the neutral voltage in the backward sweep $\left(F B S_{N}\right)$ there is a considerable increase in accuracy compared to $F B S_{N g}$. Figure 6 illustrates the error in the voltage angle calculation. Clearly, the proposed method yields more

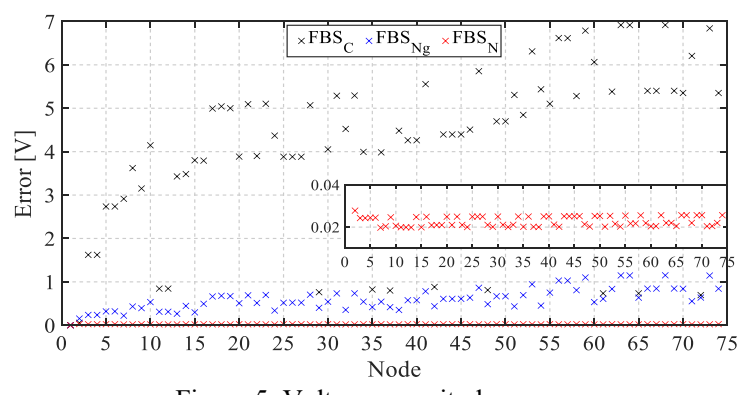

Figure 5. Voltage magnitude error

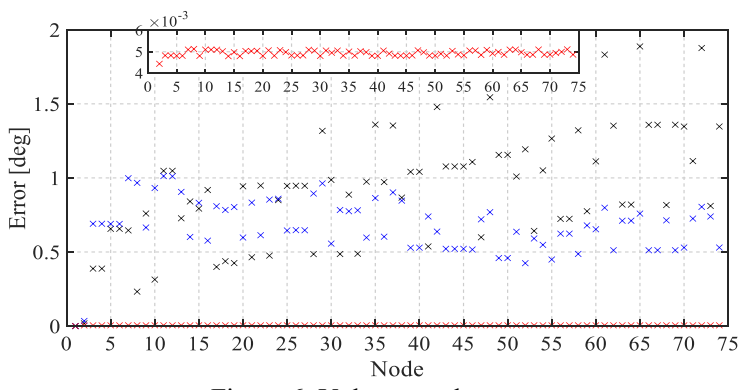

Figure 6. Voltage angle error 

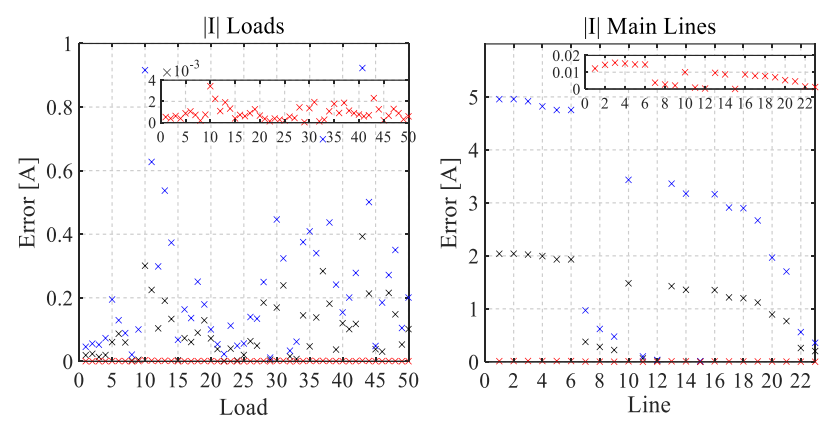

Figure 7. Current magnitude error

accurate results while $F B S_{N g}$ and $F B S_{C}$ have similar accuracy. However, in an LVDG due to the high $\mathrm{R} / \mathrm{X}$ ratio of the distribution lines and the relatively low power flow through them, the phase difference between adjacent nodes is usually negligible and therefore under this condition, all three methods have satisfactory accuracy regarding the voltage angles. In Fig. 7 the error in the current calculation for the loads and main distribution lines (distribution lines connecting the main feeder nodes) is illustrated. As before, the proposed method is significantly more accurate, especially for the currents of the main distribution lines.

In order to validate the performance of the proposed method under different operating conditions, load profiles are constructed for each load with a $30 \mathrm{~min}$ resolution and a total simulation time of 3 days. Then, all three methods are used to conduct power flow simulations based on these load profiles and in Figs. 8-9 the average voltage and current magnitude errors are illustrated. The average voltage error in each instance that a power flow is executed is calculated as,

$$
V_{e}=\sqrt{\frac{\sum_{i=1}^{N}\left(V^{i}-\hat{V}^{i}\right)^{2}}{N}}
$$

where $N$ is the total number of phase and neutral (neutral is only considered for $F B S_{N g}$ and $F B S_{N}$ methods) voltages, $V^{i}$ is the $i^{\text {th }}$ system voltage from MATLAB/Simulink and $\widehat{V}^{i}$ is the corresponding system voltage calculated with either of the three methods. The average current error is calculated in a similar fashion. From Figs. 8 and 9 it can be seen that the performance of $F B S_{C}$ is not consistent and is highly affected by the loading conditions. This is expected as in high-loaded conditions or during intense load asymmetric conditions, the neutral voltage in the system reaches significant values (due to the zero sequence currents) and the use of Kron's reduction results to an erroneous model for the distribution lines. On the

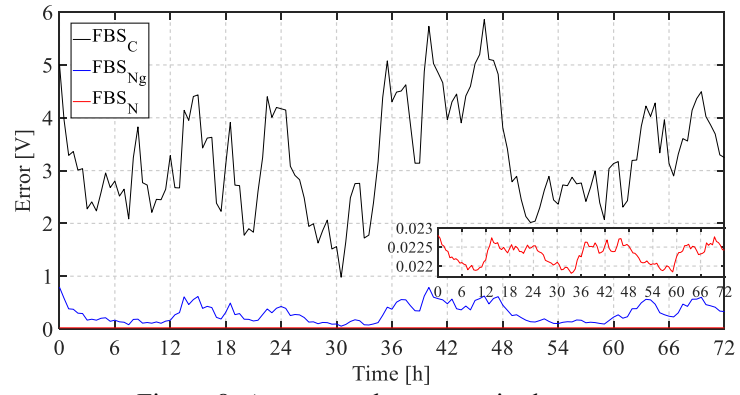

Figure 8. Average voltage magnitude error
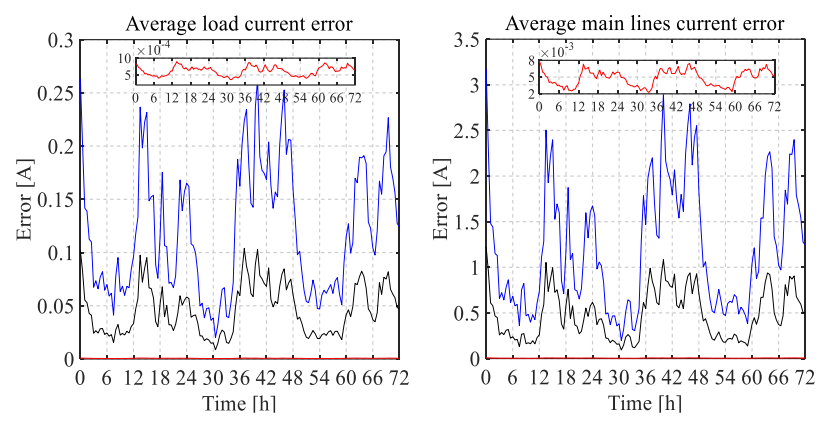

Figure 9. Average current magnitude error

TABLE I

SumMary OF Simulation RESUlts

\begin{tabular}{c|c|c|c|c|c}
\hline \hline Method & Iterations & $\begin{array}{c}\text { Average }\|\mathrm{V}\| \\
\text { error [V] }\end{array}$ & $\begin{array}{c}\text { Average } 4 V \\
\text { error [deg] }\end{array}$ & $\begin{array}{c}\text { Average } \\
\text { load }\|\mathrm{I}\| \\
\text { error [A] }\end{array}$ & $\begin{array}{c}\text { Average } \\
\text { line }\|\mathrm{I}\| \\
\text { error [A] }\end{array}$ \\
\hline \hline$F B S_{C}$ & 7.3 & 3.24 & 0.68 & 0.05 & 0.48 \\
\hline$F B S_{N g}$ & 6.2 & 0.30 & 0.53 & 0.12 & 1.22 \\
\hline$F B S_{N}$ & 7.5 & 0.02 & 0.0047 & $6.19^{*} 10^{-4}$ & $4.81 * 10^{-3}$ \\
\hline \hline
\end{tabular}

other hand, $F B S_{N}$ has almost a constant performance, regardless of the loading conditions and is more accurate at all times in comparison to $F B S_{N g}$. In Table I, a summary of the simulation results is provided along with the average number of iterations of each method. From here it can be seen that all three methods require a similar number of iterations to converge, with $F B S_{N}$ requiring a sligthly higher number due to the increased complexity. However, the significant gain in accuracy outweighs the small increase in computational burden.

\section{B. Reverse Power Flow}

An important topic in LVDGs is the possibility of reverse power flow due to high PV penetration and how it can affect their operation. However, due to the sheer size that a LVDG can have, the relatively low power flows and the importance of identifying where a reverse power flow is occurring rather than what the actual value of the power flow is, it is unnecessary and computationally inefficient to calculate the power flow in each distribution line. A simple and efficient way to determine when and where a reverse power flow is occurring is to calculate the phase difference between the voltage and current (which are the output of the power flow) of each phase of a distribution line. If this phase difference is larger than $90^{\circ}$ then a reverse power flow is occurring in a distribution line and the analysis of the power flow results can be focused around it, in order to determine how the adjacent nodes are affected. In Fig. 10 an example is provided in which the black line illustrates

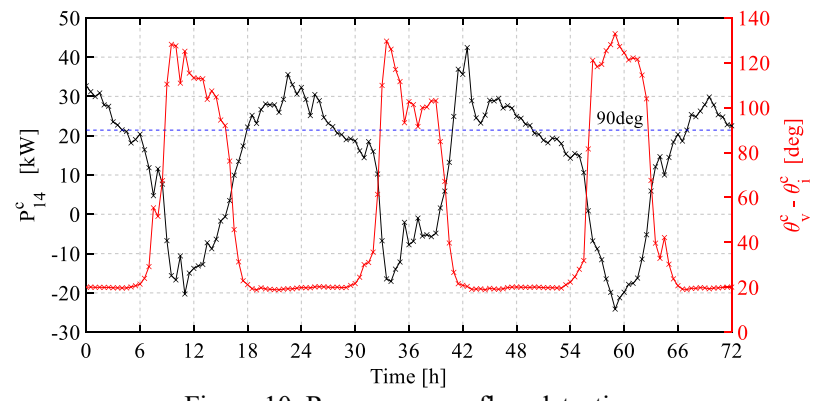

Figure 10. Reverse power flow detection 

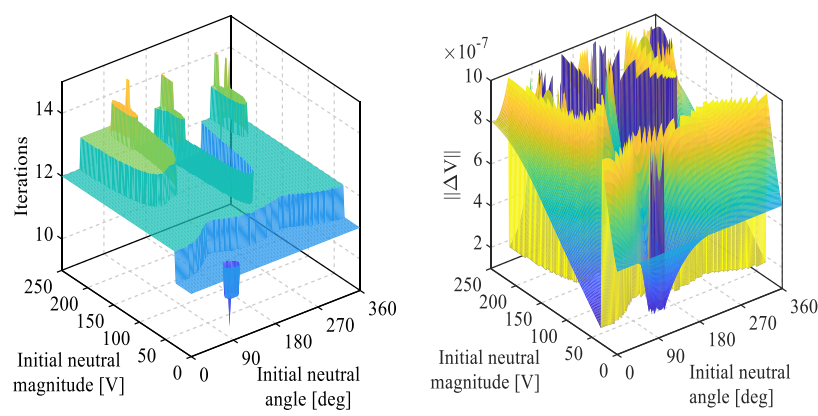

Figure 11. Convergence with different neutral initialization

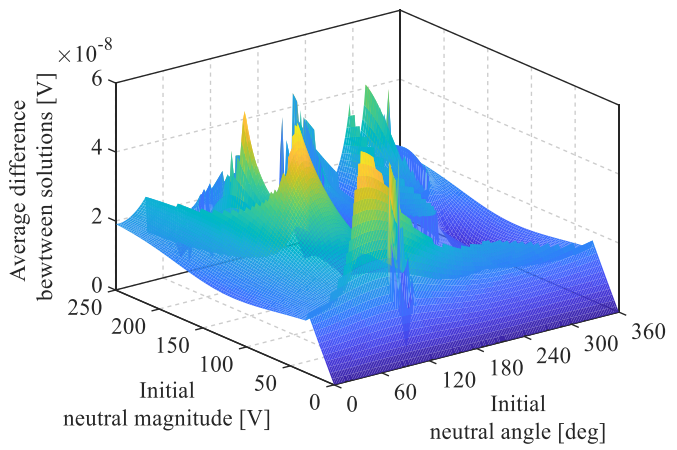

Figure 12. Comparison between solutions

the power flow of phase $c$ of the distribution line connecting nodes 9-14 while the red line illustrates the phase difference between the phase $c$ voltage and current of this line. It can be seen that when a reverse power flow occurs the phase difference is indeed larger than $90^{\circ}$. Using this method, the actual power flow can be calculated only at the distribution lines and at the time instances that a reverse power flow is detected rather than calculating all power flows for each distribution line and for the whole simulation time.

\section{Possible Multiple Power Flow Solutions}

In [15] the effect of the neutral voltage initialization on the convergence of the power flow and the possibility of multiple solutions existing is discussed for different configurations of the neutral conductor. The type of LVDG that is considered in this paper is similar to Case 5 from [15] where a radial multigrounded neutral conductor is examined. This type of system is found to have only one possible solution regardless of the initialization of the neutral voltage. In order to verify that this conclusion can be extended to radial LVDGs with a neutral conductor grounded only at the transformer substation, $F B S_{N}$ is applied in the same operating scenario as in Section III.A with however different initializations for the neutral voltage. In Fig. 11 the number of iterations needed to converge and the final voltage step are illustrated for the different initializations of the neutral voltage. From this figure it can be seen that although the number of iterations is affected by the initialization of the neutral voltage, all possible combinations achieve convergence. In Fig. 12, the average difference between the solution with neutral initialization to $0 \mathrm{~V}$ and the solutions with different neutral initialization is illustrated. From this figure it can be concluded that regardless of the initialization of the neutral voltage, $F B S_{N}$ will converge to the same solution.

\section{CONCLUSIONS}

This paper proposes a modification to the four-wire forward/backward sweep power flow method in order to take into consideration the neutral voltage for radial LVDGs with a neutral conductor grounded only at the MV-LV substation. From the numerical simulations it was seen that the proposed method achieves significantly more accurate results than relevant existing methods with a slight increase however in the required iterations due to the increased complexity. A simple and efficient method was also discussed in order to detect reverse power flows in the LVDG without having to calculate the power flow of each distribution line. Finally, the possibility of multiple power flow solutions due to the initialization of the neutral voltage was examined with a negative conclusion as all examined cases converged to the same solution.

\section{REFERENCES}

[1] International Energy Agency (2017). Status of Power System Transformation - Summary for Policy Makers. Available: https://www.iea.org/publications/freepublications/publication/StatusofPower SystemTransformation2017SummaryforPolicyMakers.pdf

[2] L. Mukwekwe, C. Venugopal and I. E. Davidson, "A Review of the Impacts and Mitigation Strategies of High PV Penetration in Low Voltage Networks," in Proc. IEEE PES-IAS Power Africa, Accra, Ghana, pp. 1-6, 2017.

[3] P. A. N. Garcia, J. L. R. Pereira, J. Carneiro, V. M. Costa and N. Martins, "Three-phase power flow calculations using the current injection method," in IEEE Trans. Power Systems, vol. 15, no. 2, pp. 508-514, May 2000.

[4] C. S. Cheng and D. Shrirmohammadi, "A three-phase power flow method for real time distribution system analysis," in IEEE Trans. Power Systems, vol. 10, no. 2, pp. 671-679, May 1995.

[5] L. R. Araujo, D. R. R. Penido, S. Carneiro Jr., J. L. R. Pereira and P. A. N. Garcia, "Comparisons between the three-phase current injection method and the forward/backward sweep method," in Int. J. Elect. Power Energy Syst., vol. 32, pp. 825-833, 2010.

[6] J. H. Teng, "A direct approach for distribution system load flow solutions," in IEEE Trans. Power Delivery, vol. 18, no. 3, pp. 882-887, July 2003.

[7] S. Elsaiah, M. Ben-Idris and J. Mitra, "Power flow analysis of radial and weakly meshed distribution networks," in Proc. IEEE PES General Meeting, San Diego, USA, pp. 1-9, 2012.

[8] H. Liu, S. Cheng, C. Huang and Y. Hou, "Unbalanced power flow calculation for low voltage distribution systems including DGs," in Proc. IEEE ISGT ASIA, Tianjin, China, pp. 1-5, 2012.

[9] P. Samal and S. Ganguly, "A modified forward backward sweep load flow algorithm for unbalanced radial distribution systems," in Proc. IEEE PES General Meeting, Denver, USA, pp. 1-5, 2015.

[10] R. M. Ciric, A. O. Feltrin and L. F. Ochoa, "Power flow in four-wire distribution networks - General Approach," in IEEE. Trans. Power Systems, vol. 18, no. 4, pp. 1283-1290, Nov. 2003.

[11] A. Kotsonias, L. Hadjidemetriou, M. Asprou and E. Kyriakides, "Performance investigation of a monitoring scheme for low voltage grids with a single grounded neutral," in Proc. IEEE PowerTech, Milan, Italy, 2019.

[12] T. H. Chen and W. C. yang, "Analysis of multi-grounded four-wire distribution systems considering the neutral grounding," in IEEE Trans. Power Delivery, vol. 16, no. 4, pp. 710-717, Oct. 2001.

[13] W. H. Kersting, Distribution System Modeling and Analysis, CRC Press, Boca Raton, FL, 2007.

[14] P. Xiao, D. C. Yu and W. Yan, "A Unified Three-Phase Transformer Model for Distribution Load Flow Calculations," in IEEE Trans. Power Systems, vol. 21, no. 1, pp. 153-159, Jan. 2006.

[15] L. Araujo, D. R. R. Penido, S. Carneiro Jr. and J. L. R. Pereira, “A study of neutral conductors and grounding impacts on the load flow solutions of unbalanced distribution systems," in IEEE Trans. on Power Systems, vol. 31, no. 5, pp. 3684-3692, Sep. 2016. 